\title{
Isolation, activity and immunological characterisation of a secreted aspartic protease, CtsD, from Aspergillus fumigatus
}

\author{
Imelda Vickers, Emer P. Reeves, Kevin A. Kavanagh, Sean Doyle* \\ National Institute for Cellular Biotechnology, Department of Biology, National University of Ireland Maynooth, Co. Kildare, Ireland
}

Received 13 November 2006, and in revised form 18 December 2006

\begin{abstract}
Aspergillus fumigatus is an opportunistic fungal pathogen that infects immunocompromised patients. A putative aspartic protease gene (ctsD; $1425 \mathrm{bp}$; intron-free) was identified and cloned. CtsD is evolutionarily distinct from all previously identified A. fumigatus aspartic proteases. Recombinant CtsD was expressed in inclusion bodies in Escherichia coli ( $0.2 \mathrm{mg} / \mathrm{g}$ cells) and subjected to extensive proteolysis in the baculovirus expression system. Activation studies performed on purified, refolded, recombinant CtsD resulted in protease activation with a $\mathrm{pH}_{\text {opt }} 4.0$ and specific activity $=10 \mathrm{U} / \mathrm{mg}$. Pepstatin A also inhibited recombinant CtsD activity by up to $72 \%$ thereby confirming classification as an aspartic protease. Native CtsD was also immunologically identified in culture supernatants and purified from fungal cultures using pepstatin-agarose affinity chromatography ( $7.8 \mu \mathrm{g}$ CtsD/g mycelia). In $\mathrm{A}$. fumigatus, semi-quantitative RTPCR analysis revealed expression of $c t s D$ in minimal and proteinaceous media only. Expression of $c t s D$ was absent under nutrient-rich conditions. Expression of $c t s D$ was also detected, in vivo, in the Galleria mellonella virulence model following A. fumigatus infection.

(C) 2006 Elsevier Inc. All rights reserved.
\end{abstract}

Keywords: ELISA; Proteases; Virulence; Insect model; Siderophores; Pepstatin, Galleria mellonella

Aspergillus fumigatus is one of the major causes of invasive aspergillosis in immunocompromised patients [1]. Although significant improvements have been forthcoming for the identification and diagnosis of A. fumigatus infection and anti-fungal therapeutic strategies, there remains a fundamental dearth of information regarding the biology of the organism [2]. The international collaborative effort directed towards the sequencing and annotation of the $A$. fumigatus genome has greatly facilitated research efforts directed towards improving our understanding of the virulence potential of the organism [3].

The degradative properties of secreted proteases have attracted much attention as potential mediators of fungal invasion in infected tissue, conidophore development and iron acquisition [4,5]. For instance, the role of aspartic proteases in particular has been extensively investigated in the yeast Candida albicans and studies have shown that these

\footnotetext{
* Corresponding author. Fax: +353 17083845.

E-mail address: sean.doyle@nuim.ie (S. Doyle).
}

key virulence factors are associated with hyphal formation, phenotypic switching, adhesion and evasion of the host immune response [6].

To date, a number of secreted proteases have been identified in A. fumigatus including a serine protease (ALP1) [7,8], a metalloprotease (MEP) ${ }^{1}[9]$ and an aspartic protease (PEP1) [10]. It appears that these enzymes alone may not play a significant role in the invasive characteristics of $A$. fumigatus following animal studies with single alp 1 , mep and pep 1 mutants and double alp 1 and mep mutants $[4,11,12]$. However, a number of uncharacterised secretory proteases remain within the genome, which may be capable of compensating for the loss of these individual enzymes by activating similar or alternative virulence pathways. C. albicans employs at least 10 secreted aspartic protease (sap) genes, and correlations between the level of proteolytic

\footnotetext{
${ }^{1}$ Abbreviations used: MEP, metalloprotease; ABPA, allergic bronchopulmonary aspergillosis; MEM, minimal essential medium; FCS, fetal calf serum.
} 
activity in vitro and virulence of the yeast have been deduced [6]. Essentially, the higher the level of proteolytic activity detected, the more virulent the Candida strain. Animal studies involving sap $1-3$ and sap4-6 mutants demonstrated the selective use of these proteases during mucosal and systemic infection $[13,14]$. This strengthens the possibility that $A$. fumigatus may also be selectively using the proteases at its disposal, depending on the stage and type of infection. It is worth noting that three classes of proteolytic enzymes have been identified in $A$. fumigatus, suggesting a wide range of activity in differing environmental conditions. However, evidence of serine, metalloprotease or cysteine protease secretion, has yet to be identified in any pathogenic strains of Candida [6].

It has been suggested that the secreted aspartic protease (PEP1) of A. fumigatus may be an allergen associated with allergic bronchopulmonary aspergillosis (ABPA) and play a lesser role in tissue invasion. PEP2, a cell wall associated aspartic protease of $A$. fumigatus may weaken the cell wall to allow for fungal cell growth [4]. Interestingly, another cell wall associated serine protease (ALP2) of A. fumigatus has been identified as a major allergen associated with allergic asthma [15], so this may suggest that the cell wall associated proteases, such as PEP2 may play an integral role in the development of allergic diseases. Alternatively, protease expression may be a prerequisite for host tissue invasion, and therefore crucial to virulence, as the regulation of these proteases appears to exhibit significant complexity, involving numerous enzymes [16,17].

In the present study, we confirm the existence of a lowabundance aspartic protease, CtsD, in A. fumigatus and demonstrate the in vitro and in vivo expression patterns of this new enzyme.

\section{Materials and methods}

\section{Bioinformatic analysis}

Preliminary sequence data were obtained from The Institute for Genomic Research website at http:// www.tigr.org. The unannotated genome of A. fumigatus was interrogated to identify open reading frames corresponding to novel aspartic proteases. One orf was identified, which was subsequently termed ctsD (GenBank Accession No.: EAL90084). Sequence homologies were determined using the BLAST algorithm (http:// www.ncbi.nlm.nih.gov/blast/bl2seq/bl2.html) and ClustalW (http://www.ebi.ac.uk/clustalw/). The presence of signal peptides was identified using the SignalP program (http:// www.cbs.dtu.dk/). The MEROPS peptidase database (http:/ /merops.sanger.ac.uk/) was used to facilitate cts $D$ analysis [18].

\section{Fungal DNA isolation and PCR amplification}

Aspergillus fumigatus strain ATCC 26933 was grown (250 ml cultures) at $37^{\circ} \mathrm{C}$ in minimal essential medium
(MEM) (containing 5\% (v/v) fetal calf serum (FCS)) for 2 days. Genomic DNA was isolated as previously described [19]. A total of $5 \mu \mathrm{l}$ of genomic DNA was used in all PCRs with $10 \mathrm{mM}$ Tris- $\mathrm{HCl}, \mathrm{pH} 9.0,50 \mathrm{mM} \mathrm{KCl}, 0.1 \%$ (v/v) Triton X-100, $1.5 \mathrm{mM} \mathrm{MgCl} 2,200 \mu \mathrm{M}$ of each dNTP, $1.0 \mu \mathrm{M}$ cts $D$-forward (5'-GAGAAGAGCTATGCATCTCTTAC AATGTCTACTTTCG-3') and $c t s D$-reverse primer $\left(5^{\prime}-\mathrm{G}\right.$ AGACCCGGGCACCAACACCATGCAAAGAAGTG-3') in a total volume of $49.5 \mu 1$. PCRs comprised an initial step of $300 \mathrm{~s}$ denaturation at $95^{\circ} \mathrm{C}$, followed by 30 cycles consisting of $60 \mathrm{~s}$ at $95^{\circ} \mathrm{C}, 30 \mathrm{~s}$ at $55^{\circ} \mathrm{C}, 90 \mathrm{~s}$ at $72^{\circ} \mathrm{C}$ and finally for $360 \mathrm{~s}$ at $72^{\circ} \mathrm{C}$. Amplicons were analysed by agarose gel electrophoresis and visualised using an 'Eagle-Eye II' digital still video system (Stratagene, CA, USA). DNA sequence analysis was carried out on a commercial basis (MWG Biotech, Germany).

\section{Cloning and expression of the aspartic protease gene, ctsD}

The $c t s D$ amplicon was cloned into $\mathrm{pProEx}^{\mathrm{TM}}$ expression vector and baculovirus recombinants of $c t s D$ were also generated according to the manufacturer's instructions (Invitrogen). pProEx:cts $D$, the expression vector containing $A$. fumigatus ctsD, was transformed into Escherichia coli according to the manufacturer's guidelines (Invitrogen). Protein expression was induced by the addition of isopropyl- $\beta$-D-thiogalactopyranoside (IPTG; $0.6 \mathrm{mM}$ final concentration) and monitored by SDS-PAGE, Western blot analysis ( $\mathrm{His}_{6}$ fusion tag) and MALDI-TOF mass spectrometry [19].

\section{Recombinant CtsD purification, activity and immunological analysis}

Recombinant CtsD was purified under denaturing conditions using Ni-NTA metal-chelate affinity chromatography in the presence of $6 \mathrm{M}$ guanidine- $\mathrm{HCl}$ [20]. Purified recombinant CtsD $(250 \mu \mathrm{g} / \mathrm{ml})$ in $50 \mathrm{mM}$ sodium carbonate, $\mathrm{pH}$ 9.4, containing $6 \mathrm{M}$ guanidine- $\mathrm{HCl}$ was serially dialysed into $50 \mathrm{mM}$ sodium carbonate, $\mathrm{pH} 9.4$, containing $8 \mathrm{M}$ urea, then $4 \mathrm{M}$ urea, followed by dialysis into $50 \mathrm{mM}$ sodium carbonate buffer, $\mathrm{pH}$ 9.4, to facilitate solubilisation. Protease activity was quantified using bovine hemoglobin as substrate using a modified protocol ( $2 \mathrm{~h}$ incubation) to that previously described [21]. One unit of activity was defined as $A_{280 \mathrm{~nm}}=0.1$ observed after a two hour incubation at $37^{\circ} \mathrm{C}$ ( $\left.\mathrm{pH} 4.0\right)$. Pepstatin inhibition studies were carried out as previously described [22]. Rabbit immunisation $(n=5$ occasions) was performed over a period of 4 months using purified recombinant $\mathrm{CtsD}(50 \mu \mathrm{g}$ per immunisation) as antigen source. Rabbit CtsD antibody was purified by Protein-A-Sepharose ${ }^{\mathrm{TM}}$ affinity chromatography (Amersham Biosciences). Pre-immune rabbit antisera was also purified in this manner. Western blot detection of both native and recombinant $\mathrm{CtsD}$ was performed using immune antisera $(1 / 2000)$ and goat anti-rabbit IgG-horseradish peroxidase conjugate $(1 / 1000-1 / 15000)$ using either diaminobenzidine 
(Sigma-Aldrich) or enhanced chemiluminescent (ECL) detection (Pierce Biotechnology).

\section{Native CtsD protein analysis and purification}

Aspergillus fumigatus culture supernatants (24-96h; MEM and MEM + 5\% (v/v) FCS) were collected and subjected to immunological analysis using $\mathrm{CtsD}$ antisera. CtsD was purified from MEM culture supernatants $(72 \mathrm{~h} ; 250 \mathrm{ml})$ on pepstatin-agarose affinity resin (Pierce Biotechnology). The column $(5 \mathrm{ml})$ was washed to remove unbound proteins using phosphate-buffered saline (PBS) and fractions $(0.5 \mathrm{ml})$ were eluted using $20 \mathrm{mM}$ Tris containing $450 \mathrm{mM}$ $\mathrm{NaCl}, \mathrm{pH}$ 7.9. Enzyme activity was determined as described above.

\section{CtsD-specific competitive ELISA}

The presence of CtsD in both MEM and MEM + 5\% (v/v) FCS culture supernatants was determined by competitive ELISA. Relevant A. fumigatus culture supernatants were pre-incubated at room temperature for $30 \mathrm{~min}$ at $200 \mathrm{rpm}$ with CtsD antisera (1/1000 dilution). Specimens $(100 \mu 1$ each) were loaded, in duplicate, onto 96-well microtitre plates pre-coated with recombinant CtsD antigen $(0.2 \mu \mathrm{g} / \mathrm{well})$ and incubated for $1 \mathrm{~h}$ at room temperature. Plates were washed twice with PBS- $0.05 \%(\mathrm{v} / \mathrm{v})$ Tween 20, protein A-horseradish peroxidase conjugate $(100 \mu \mathrm{l} / \mathrm{well})$ added and incubated for $1 \mathrm{~h}$ at room temperature. After washing, tetramethylbenzidine substrate ( $100 \mu \mathrm{l} /$ well) was added incubated for $10 \mathrm{~min}$ and the reaction was stopped by the addition of $100 \mu \mathrm{l}$ of $1 \mathrm{~N}$ sulphuric acid per well. The absorbance was determined at $450 \mathrm{~nm} / 630 \mathrm{~nm}$ and the concentration of CtsD in media supernatants was determined from a standard curve of 0 $10 \mu \mathrm{g} / \mathrm{ml}$ recombinant CtsD.

\section{ctsD gene expression in culture}

Aspergillus fumigatus conidia were inoculated into (i) Sabouraud media, (ii) 5\% (v/v) FCS in MEM, (iii) $10 \%$ (v/v) FCS in MEM, and (iv) 20\% (v/v) FCS in MEM, respectively. Cultures were harvested at defined time-points (24, 48, 72 and $96 \mathrm{~h}$ ) and total $A$. fumigatus RNA was isolated using a TRI ${ }^{\circledR}$ reagent method (Sigma-Aldrich) and DNase I-treated. Equivalent amounts $(0.5 \mu \mathrm{g})$ of RNA were used to generate cDNA at each time point, in addition to carrying out parallel control RT-PCR using calm primers as the housekeeping gene [23]. cDNA was synthesised according to standard procedures (Invitrogen) and $1 \mu l$ of each cDNA sample was introduced into PCR reactions as described above using PCR primers: forward (5'-GAGAAGGCCT TCAAATGGTGTCTCTTTAACGTTGG-3') and reverse (5'-GAGAGGTACCCACCAACACCATGCAAAGAA GTG-3'). As calm DNA contains introns, a larger amplicon was expected for the genomic DNA relative to the cDNA amplicon.

\section{Virulence model}

Experimental approaches were developed (i) to determine if $c t s D$ was expressed during infection and (ii) to assess the functional role of CtsD, using the Galleria mellonella insect model [24]. First, G. mellonella larvae ( $=200$ ) were inoculated into the hind pro-leg with $5 \times 10^{5}$ A. fumigatus conidia in $20 \mu \mathrm{l}$ (per larva) followed by total RNA extraction at $0,24,48,72$ and $96 \mathrm{~h}$ time-points (50 larvae/RNA extraction). ctsD gene expression was assessed using RT-PCR as described above. Second, $G$. mellonella larvae ( $n=20$ per group) were inoculated as above with either (i) A. fumigatus conidia $\left(10^{7}\right.$ per larvae), (ii) co-inoculated with $20 \mu \mathrm{g}(2 \mu \mathrm{g} / \mu \mathrm{l})$ of rabbit antiCtsD IgG and $10^{7}$ A. fumigatus conidia, (iii) pre-immune rabbit IgG $(20 \mu \mathrm{g}(2 \mu \mathrm{g} / \mu \mathrm{l}))$ with $10^{7}$ conidia or (iv) PBSonly inoculation (negative control). The survival rate of the G. mellonella larvae was determined at $72 \mathrm{~h}$. Statistical analysis was carried out using the ANOVA one-way analysis of variance test.

\section{Results and discussion}

\section{Phylogenetic analysis of CtsD and related aspartic proteases}

Using a bioinformatic approach, a unique aspartic protease (CtsD), demonstrating significant identity (58\%) to an unnamed protein product of Aspergillus oryzae (GenBank Accession No.: BAE59370), 52\% identity to an $A$. nidulans hypothetical protein and $48 \%$ identity to aspartyl protease 4 (PEP4) of Coccidioides posadasii, was identified following BLAST analysis of the unannotated A. fumigatus genome. CtsD was also classified as a subfamily A1A aspartic protease following MEROPS analysis [18]. CtsD formed no grouping with any other characterised or putative aspartic protease of $A$. fumigatus (at least seven in total; data not shown). Moreover, amino acid sequence identity of $30 \%$ was observed with only one putative cellular aspartic protease in A. fumigatus (GenBank Accession No.: XP_754479). CtsD exhibited $28 \%$ and $29 \%$ identity to A. fumigatus PEP1 [10] and PEP2 [4], respectively. CtsD exhibited 24\% identity to a previously unidentified putative aspartic protease (OpsB; [3] in this organism. An OpsB homologue is also present in $A$. oryzae. Identity (32\%) was also shared between CtsD and preprocathepsin D of Dictyostelium discoideum [25]. Phylogenetic analysis of CtsD with aspartic proteases of Aspergillus niger (PEPE; [26]) and C. albicans (SAP2 and SAP6) demonstrated that these proteases are unlikely to be sister taxa of CtsD (Fig. 1). A 19 amino acid signal peptide was also identified using the SignalP program, suggesting a secretory pathway for CtsD production, however, no propeptide cleavage site was predicted. A conserved aspartic protease DTG-motif was present in the active site of all analysed sequences (data not shown). 


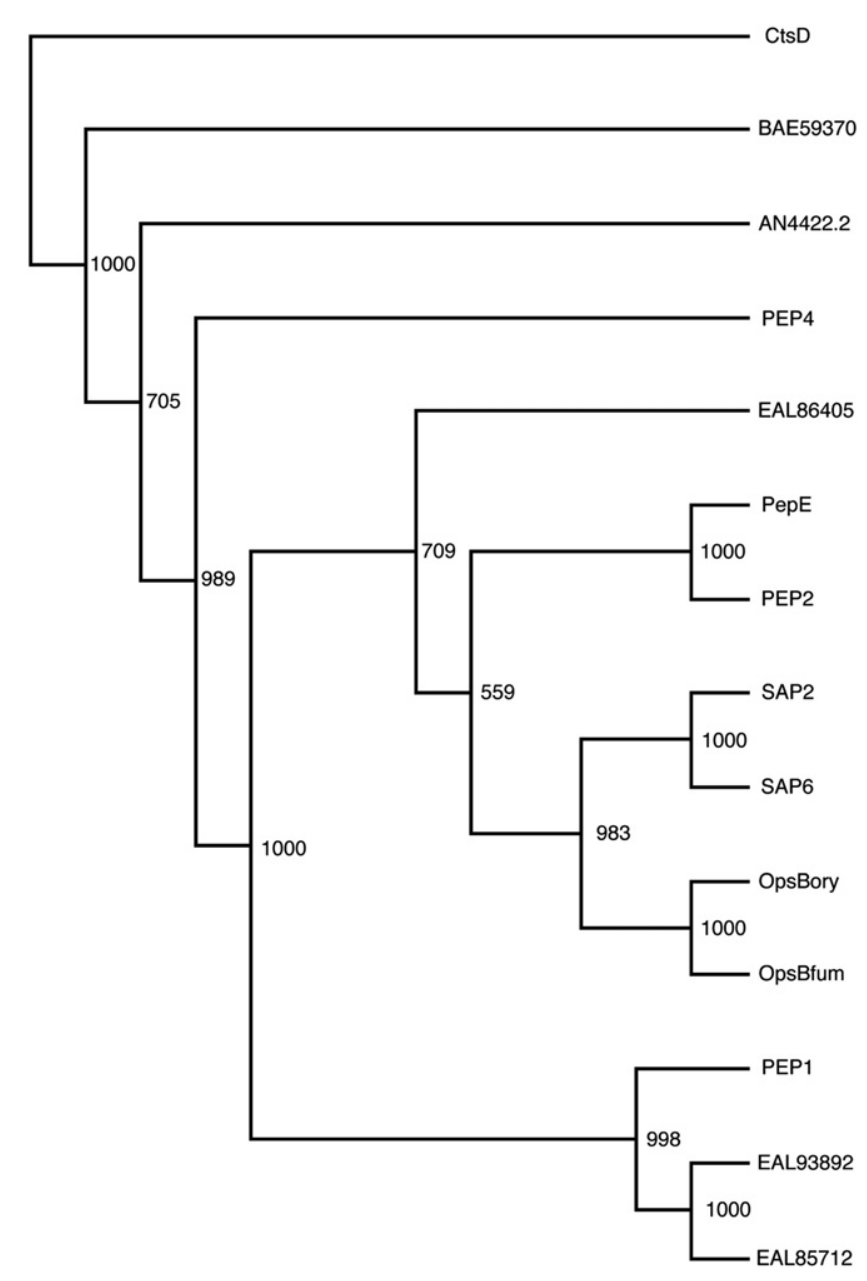

Fig. 1. Phylogenetic relationship between $A$. fumigatus $\mathrm{CtsD}$ and selected fungal aspartic proteases (actual and putative). CtsD is most closely related to putative aspartic proteases from related fungal species but distinct from previously identified enzymes (PEP1 and 2) in A. fumigatus. Aspartic proteases: CtsD (GenBank Accession No. (GAN): EAL90084), A. oryzae (putative) (PAPory; GAN: BAE59370), A. nidulans (putative) (PAPnid; GAN: AN4422.2), C. posadasii PEP4 (GAN: ABA54909), A. fumigatus (putative) (PAPlfum; GAN: EAL86405), A. niger (PEPE; GAN: AAA0876), A. fumigatus PEP2 (GAN: CAA75754), C. albicans (SAP2 and SAP6; GAN: XP_711047 and Z30192, respectively), A. oryzae (putative) (OpsBory; GAN: BAC00848), A. fumigatus (putative) (OpsB; GAN: XM_742449), A. fumigatus PEP1 (GAN: EAL91286), A. fumigatus (putative) (PAP2fum; GAN: EAL93892) and A. fumigatus (putative) (AP1fum; GAN: EAL85712).

\section{Expression of recombinant CtsD in E. coli}

Following PCR optimisation, the entire open reading frame of $c t s D$ was isolated, yielding a product of $1425 \mathrm{bp}$. Following DNA sequence analysis it was subsequently inserted into a pProEx expression vector. Protein expression plasmid pProEx:cts $D$, consisting of the vector pProEx$\mathrm{Htb}^{\mathrm{TM}}$ containing the entire open reading frame of $c t s D$ was transformed into E. coli XL1-Blue and expression induced at $0.6 \mathrm{mM}$ IPTG (Fig. 2a). The resultant recombinant protein $(58 \mathrm{kDa})$ was highly insoluble and present in the cell pellet as determined by SDS-PAGE and Western blot analysis using monoclonal antibody reactivity against a $\mathrm{His}_{6}$ tag present on recombinant CtsD (Fig. 2b). Recombinant CtsD was purified using differential extraction and Ni-NTA metal-chelate affinity chromatography, with a yield of approximately $0.2 \mathrm{mg} / \mathrm{g}$ of $E$. coli and to a purity greater than $95 \%$ as judged by SDS-PAGE and Western analysis (Fig. 2c and d). Peptides (following tryptic digestion of CtsD) were identified by MALDI-TOF mass spectrometry [19] which corresponded to the theoretical amino acid sequence of CtsD whereby $7 / 42$ peptides $(22 \%$ sequence coverage) was observed. Recombinant PEP2 has been successfully expressed in, and secreted from, the yeast Pichia pastoris [21].

\section{Immunological and activity analysis of recombinant CtsD}

The antisera generated against recombinant CtsD was highly reactive (detection limit: $12 \mathrm{ng}$ recombinant $\mathrm{CtsD}$ ) and distinct bands were visible following Western blot analysis (Fig. 2e). The observed immunogenicity of CtsD is not unexpected. Indeed, an allergenic role for fungal proteases has been previously proposed for a number of proteases PEP1 [10], ALP2 [15] and it is thought that they may play an important role in conditions such as allergic asthma or ABPA. In an attempt to express soluble and functional CtsD in a eukaryotic system for activity analysis, expression of CtsD was also undertaken using the baculovirus expression system in Spodoptera frugiperda $\left(\mathrm{Sf}_{9}\right)$ insect cells. The antisera raised against the E. coli-expressed recombinant CtsD was used to detect CtsD expression. Although intracellularly located recombinant CtsD was evident at different molecular masses (30$60 \mathrm{kDa}$; (Fig. 2f and g)) and $80 \mathrm{kDa}$ (not shown), possibly due to glycosylation or proteolytic processing, protein insolubility was again observed. At least $6 \mathrm{~N}$-glycosylation sites were predicted using NetNGlyc 1.0 (http://www.cbs.dtu.dk), with the highest support for an $\mathrm{N}$-glycosylation site at amino acid positions 301 and 304 . Only one $O$-glycosylation site at position 421 of the native protein was predicted using the DictyOGlyc 1.1 program. Baculovirus-expressed recombinant CtsD was not secreted (data not shown).

Consequently, activity and inhibitor analysis was carried out on refolded, E. coli-expressed, CtsD which obviated the requirement to express the protein in an alternative expression system (e.g., A. oryzae or Pichia pastoris). It can be seen from Fig. 3a that optimal CtsD enzyme activity was evident at $\mathrm{pH} 4.0$ and was 2.5 times greater than that observed at $\mathrm{pH} 3.0$ as determined by the hemoglobin proteolysis assay (using $5 \mu \mathrm{g}$ recombinant $\mathrm{CtsD}$ per assay). Moreover, the specific activity of recombinant CtsD was $10 \mathrm{U} / \mathrm{mg}$ under optimal assay conditions ( $\mathrm{pH} 4.0)$. The narrow $\mathrm{pH}$ range over which activity was evident contrasts markedly with the wide activity range ( $\mathrm{pH} 2-7)$, with $\mathrm{pH}_{\text {opt }}$ at 2.5-3.0, observed for PEP2 from A. fumigatus [21]. Furthermore, the localisation of PEP2 to the cell wall fraction and absence from culture supernatants indicates that both PEP2 and CtsD play distinct roles in A. fumigatus. Recombinant CtsD activity was inhibited by up to $72 \%$ in the presence of $14 \mu \mathrm{M}$ Pepstatin A (Fig. 3b). The observation of 


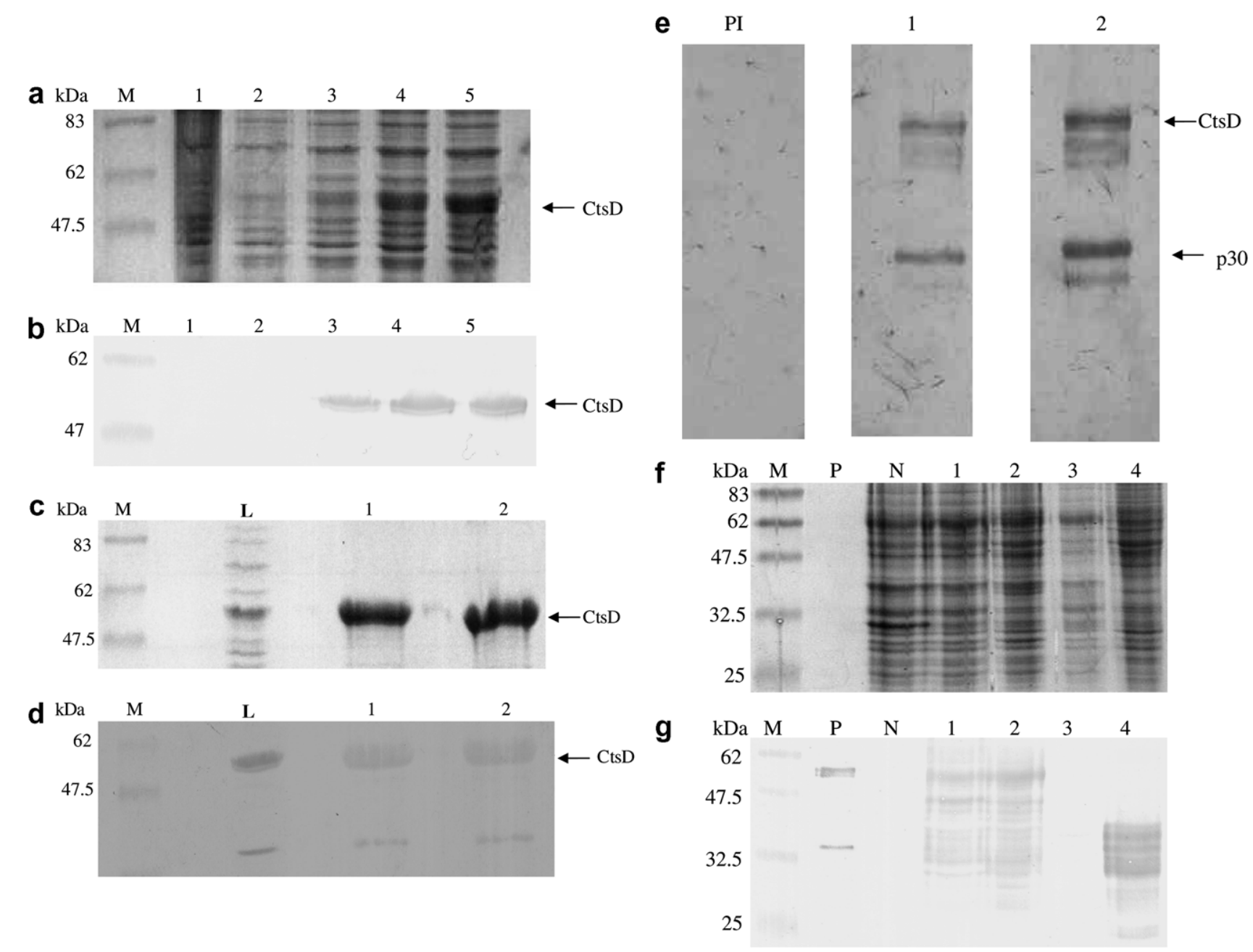

Fig. 2. SDS-PAGE and Western blot analysis of CtsD expressed in E. coli and the baculovirus expression system. (A) Coomassie stained SDS-PAGE gel of induced $E$. coli expressing CtsD. (B) Western blot of recombinant $E$. coli expressing CtsD. The Western blot was probed with $1 / 500$ dilution of anti-His 6 monoclonal antibody, 1/1000 dilution of anti-mouse IgG-horseradish peroxidase. (Lane M) Protein molecular mass marker. (Lane 1) Untransformed XL1 Blue E. coli. (Lane 2) Uninduced XL-1 Blue E. coli. (Lanes 3-5) XL-1 Blue E. coli, 1, 2 and 3 h post-induction with IPTG, respectively. A band representative of recombinant CtsD is present in each of these tracks. SDS-PAGE (C) and Western blot (D) of purified recombinant CtsD following differential extraction and Ni-NTA metal-chelate affinity chromatography. Western blot of purified batches probed with 1/500 dilution of monoclonal anti- His ${ }_{6}, 1 /$ 1000 dilution of goat anti-mouse IgG-HRP, and diaminobenzidine $/ \mathrm{H}_{2} \mathrm{O}_{2}$ substrate system. (Lane M) Protein marker. (Lane L) E. coli cell lysate containing recombinant CtsD. (Lanes 1-2) Purified CtsD - Batches 1 and 2, respectively. (E) Western blot analysis of rabbit polyclonal anti-CtsD sera reactivity against recombinant CtsD. Recombinant CtsD (1.5 $\mu \mathrm{g} /$ track) was probed (1/100 dilution) with respective antisera and 1/1000 dilution of protein A-horseradish peroxidase conjugate. (Lane PI) Rabbit pre-immune sera. (Lanes 1 and 2) Rabbit anti-CtsD sera bleed 1 and final bleed, respectively. Immunoreactive CtsD fragments (p30) of lower molecular mass $(30 \mathrm{kDa})$ are also visible. SDS-PAGE (F) and Western blot analysis (G) of CtsD recombinant baculovirus clones (recombinants 1-4). (Lane M) Protein marker. (Lane P) E. coli-expressed CtsD (0.1 $\mu$ g). (Lane N) Sf9 insect cells only. (Lanes 1-4) CtsD recombinants $1-4$, respectively. Western blots were probed with a 1/200 dilution of anti-CtsD IgG, followed by a 1/1000 dilution of goat anti-rabbit IgG-horseradish peroxidase conjugate/diaminobenzidine. Recombinants 1 and 2 demonstrated a similar expression pattern, with expression detected at $58 \mathrm{kDa}$. The lower band of approximately $45 \mathrm{kDa}$ may have been a mature and processed form of CtsD. Recombinant 4 was evident at $35 \mathrm{kDa}$, and may represent a truncated form of CtsD.

Pepstatin A sensitivity confirmed that CtsD is an aspartic protease as has been previously demonstrated for both PEP1 and PEP2, although complete activity inhibition was observed for PEP1 and PEP2 [4,21]. This difference may be related to the distinct evolutionary nature of CtsD versus PEP1/PEP2 (Fig. 1).

Reichard et al. [27] have also reported the identification of four secreted proteases (Sedolisin A-D) from A. fumigatus which are distinct acidic proteases relative to CtsD. Sedolisin $\mathrm{A}$, an endoprotease, exhibits a $\mathrm{pH}_{\text {optimum }} 5.5(\mathrm{pH}$ range 3.0-6.5), while Sedolisin $\mathrm{B}-\mathrm{D}\left(\mathrm{pH}_{\text {optima }}(\mathrm{pH}\right.$ range): 6.0 (3.0-7.0), $6.0(3.0-7.0)$ and $5.0(1.5-6.0)$, respectively) exhibit tripeptidyl peptidase activity only.

\section{Immunological and activity analysis of native CtsD}

Analysis of A. fumigatus native proteins following pepstatin-agarose affinity chromatography of MEM culture supernatants $(72 \mathrm{~h})$ demonstrated the presence of a protein of identical molecular mass to recombinant $\mathrm{CtsD}$, in addi- 

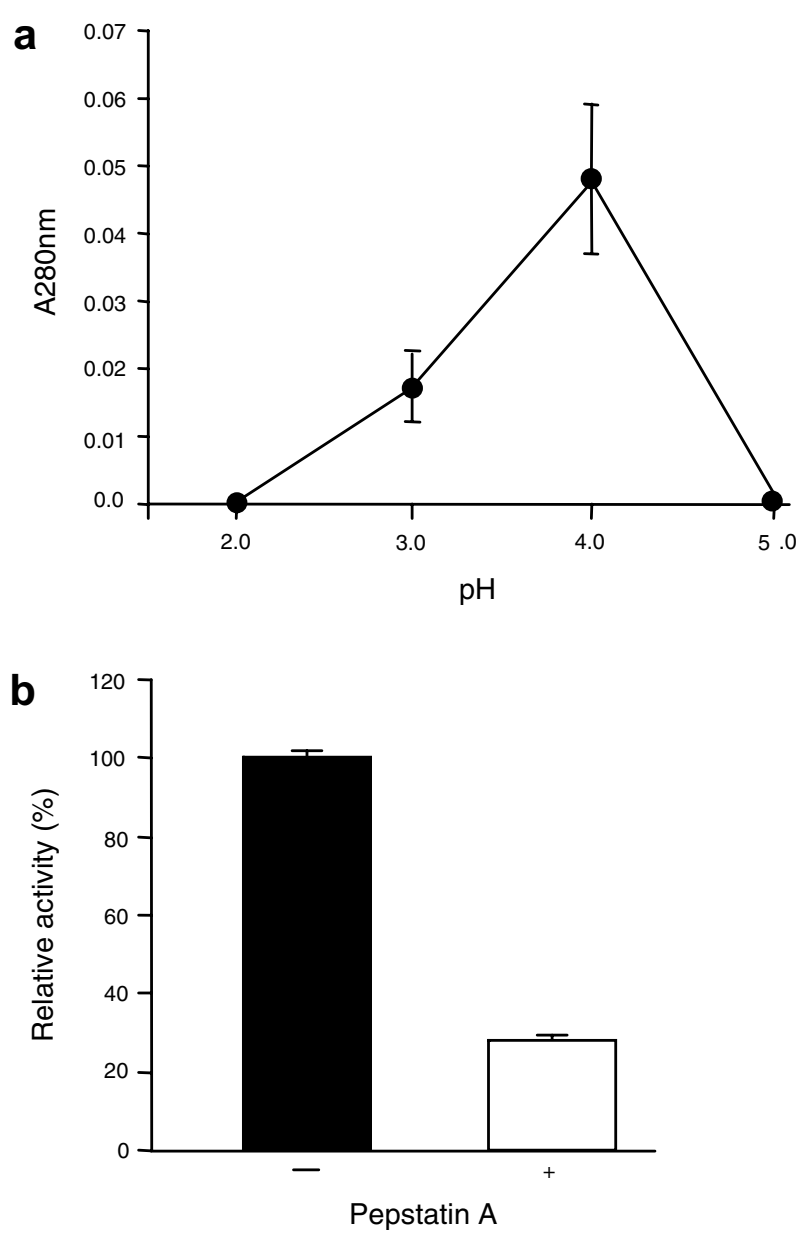

Fig. 3. (a) Activity analysis of activated recombinant CtsD. Optimal activity was obtained at $\mathrm{pH} 4.0$ according to the hemoglobin assay. No activity was detected at either $\mathrm{pH} 2.0$ or 5.0. All assays were performed in duplicate and data are presented as means \pm SE. (b) Inhibition of recombinant CtsD activity with Pepstatin A. Column 1 represents the activity detected for recombinant $\mathrm{CtsD}$ alone and column 2 represents the activity detected following incubation of the aspartic protease inhibitor Pepstatin A $(0.014 \mu \mathrm{M})$, using hemoglobin as substrate. All assays were performed in duplicate. Recombinant CtsD activity was inhibited (72\%) in the presence of Pepstatin A.

tion to a $40 \mathrm{kDa}$ protein (p40; Fig. 4a, fraction 3). Immunoreactivity was also evident at a molecular mass coincident with that of recombinant $\mathrm{CtsD}$ which confirms that native CtsD is secreted by A. fumigatus (Fig. 4b). The lower molecular mass protein, p40, was also detected using antiCtsD antibody (Fig. 4b) and may represent a degradation product or a proteolytically processed, active form of CtsD. In addition, aspartic protease activity $(28 \mathrm{U})$ was also detected in these column fractions. Using a CtsD competitive ELISA, CtsD $(3.65 \mu \mathrm{g} / \mathrm{ml})$ was detected in acetone-precipitated $72 \mathrm{~h}$ MEM supernatants, which was significantly higher then enzyme levels observed at any other time-point. CtsD levels of approximately $3 \mu \mathrm{g} / \mathrm{ml}$ were also detected in $96 \mathrm{~h} \mathrm{MEM}+5 \%$ (v/v) FCS culture supernatants. These observations suggest that CtsD is secreted from A. fumigatus as a zymogen. Similarly, human procathepsin D $(52 \mathrm{kDa})$ is proteolytically processed to a $48 \mathrm{kDa}$ protein

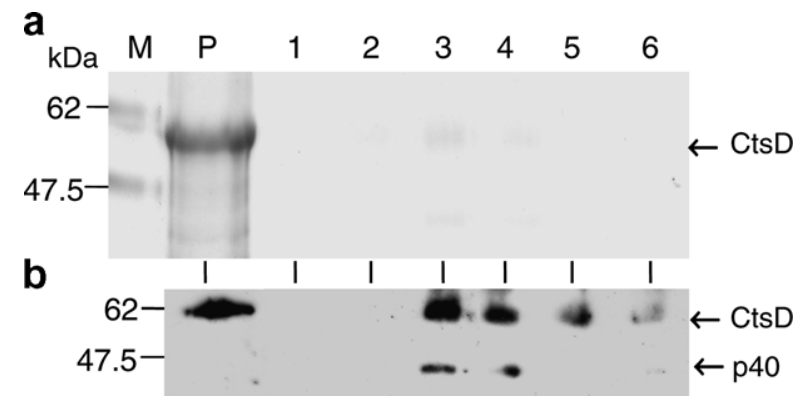

Fig. 4. SDS-PAGE and Western blot analysis of native CtsD from purified from A. fumigatus culture supernatant ( $72 \mathrm{~h}$ post-inoculation) following pepstatin-agarose affinity chromatography. (a) Coomassie blue stained SDS-PAGE analysis of purified fractions. (Lane M) Protein marker. (Lane P) Recombinant CtsD from E. coli. (Lanes 1-6) Column fractions 1-6, respectively. (b) Western blot analysis of purified fractions. The Western blot was probed with a 1/200 dilution of anti-CtsD sera, 1/ 1000 dilution of protein A-horseradish peroxidase conjugate and an enhanced chemiluminescent (ECL) substrate detection system was employed. (Lane M) Protein marker. (Lanes 1-8: Column fractions 1-6, respectively. (Lane P) Recombinant CtsD from E. coli. Protein p40 was evident in fraction 3 following pepstatin-agarose chromatography.

during transport to, or arrival at, the lysosome [28]. The fact that the zymogen form of native CtsD was evident after purification may be due to the $\mathrm{pH}$ employed for affinity chromatography.

The extent and pattern of protein secretion by A. fumigatus (strain ATCC 46645) has been demonstrated to be dependent on the media used for fungal culture, although it is possible that selective concentration of culture supernatants is necessary for optimal secreted protein detection [29]. These authors used direct $\mathrm{N}$-terminal sequence and MALDI-TOF mass spectrometry analysis to determine the presence of 5 proteins (mitogillin, PEP1, chitosanase $(\mathrm{CsnB})$, chitinase (ChiB1) and a ( $\beta 1-3)$ endoglucanase) in A. fumigatus culture supernatants, primarily in Aspergillus minimal media (AMM). Schwienbacher et al. [29] noted that no significant protein secretion was detectable after growth in Hepes-buffered RPMI 1640 cell culture medium. Thus, the affinity chromatographic approach adopted to purify native CtsD from A. fumigatus MEM culture supernatants may therefore be essential to identify low-abundance secreted proteins from $A$. fumigatus.

\section{Differential expression of ctsD under alternative culture conditions}

Total RNA was extracted from A. fumigatus grown under differential culture conditions, purity assessed, and quantitation achieved, by UV spectrophotometry and rRNA visualisation (Fig. 5a). Control RT-PCR analysis using calm primers confirmed the absence of genomic DNA as determined by the smaller amplicon size of the calm cDNA amplicon relative to the corresponding genomic amplicon (data not shown) [23]. ctsD does not contain introns as the same amplicon size was obtained for amplicons representative of cDNA and genomic DNA. Expression of $c t s D$ is not constitutive and is dependent on the 
a
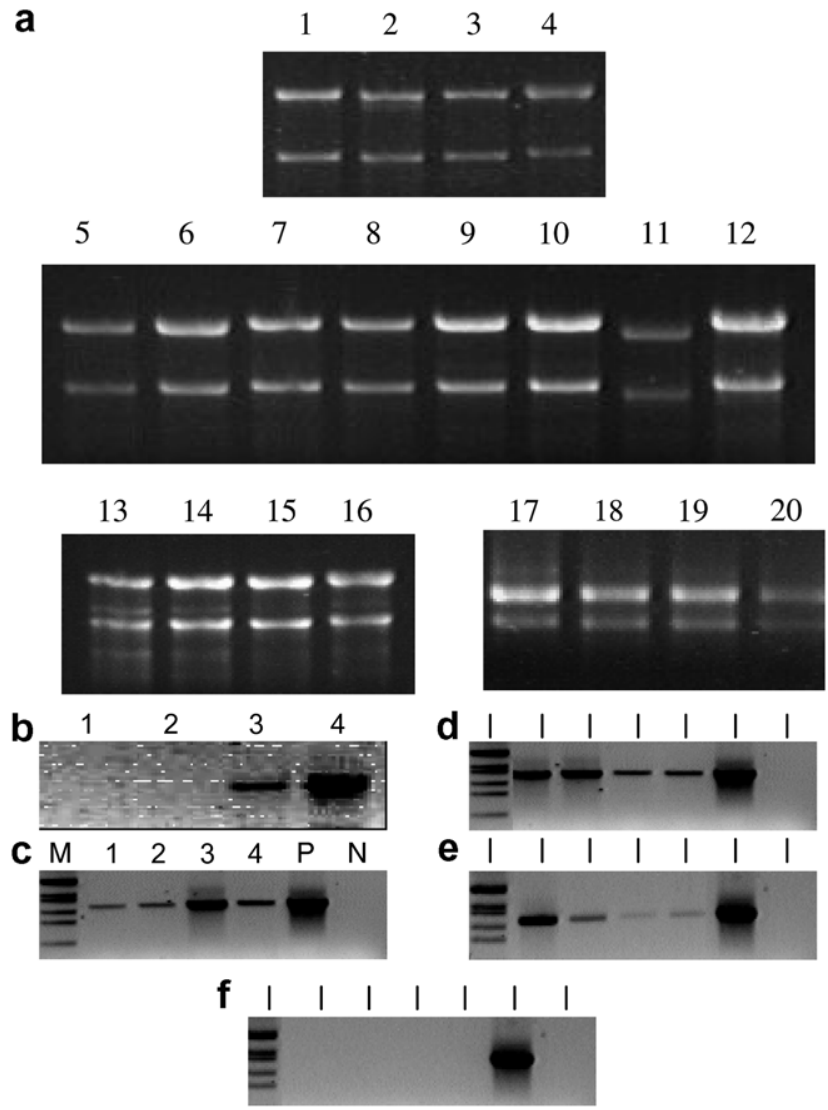

Fig. 5. Expression analysis of ctsD expression between 24 and $96 \mathrm{~h}$ under various culture conditions. (a) Quantitation of intact RNA from A. fumigatus: (1-4) MEM only, (5-8) MEM + 5\% (v/v) FCS, (9-12) MEM + 10\% (v/v) FCS, (13-16) MEM + 20\% (v/v) FCS and (17-20) Sabouraud media, respectively. (b-f) RT-PCR analysis of ctsD expression between 24 and $96 \mathrm{~h}$ under various culture conditions. (b) Lanes 1-4: cts D cDNA generated from A. fumigatus 24, 48, 72 and $96 \mathrm{~h}$ following culture in MEM only, (c) MEM + 5\% (v/v) FCS, (d) MEM + 10\% (v/v) FCS, (e) MEM + 20\% (v/ v) FCS and (f) Sabouraud media, respectively. (Lane P) $c t s D$ genomic DNA. (Lane N) Negative control. (Lane M) DNA marker. Expression of cts $D$ is apparent at 72 and $96 \mathrm{~h}$ culture time-points in MEM alone and at all time-points when FCS was present. No cts $D$ expression detected in $A$. fumigatus cultured in Sabouraud media over a $24-96 \mathrm{~h}$ time period.

growth medium used for fungal culture. In buffered cultures, expression of $c t s D$ by RT-PCR, was evident at 72 and $96 \mathrm{~h}$ only following culture of A. fumigatus in MEM medium alone (Fig. 5b). Further analysis of $c t s D$ expression revealed constitutive expression at 24, 48, 72 and $96 \mathrm{~h}$ in proteinaceous media (i.e., MEM containing $5 \%, 10 \%$ and $20 \%(\mathrm{v} / \mathrm{v})$ FCS, respectively) (Fig. 5c-e) but not in nutrient rich, non-proteinaceous, Sabouraud media at any time point (Fig. 5f) and is suggestive of a role for CtsD in the release of iron from iron-binding proteins.

Interestingly, Gifford et al. [5] have shown that induction of protease secretion occurs in A. fumigatus cultured in the presence of human or fetal bovine serum (FBS) and that it may, in part, contribute to the release of iron from ironbinding proteins by proteolysis. These authors noted that iron levels were undetectable in MEM and that FBS contained $20 \mu \mathrm{M}$ iron (equivalent to $1-4 \mu \mathrm{M}$ iron in $5-20 \%$ (v/ v) FCS). Expression of ctsD was absent in Sabouraud media which only contains $5 \mathrm{nM}$ iron (data not shown), thus it seems unlikely that $\mathrm{Cts} \mathrm{D}$ plays a role in iron acquisition in the absence of iron-binding proteins such as transferrin. It is notable that the assay conditions employed by Gifford et al. to assess protease activity ( $\mathrm{pH} 7.5)$ would have precluded detection of aspartic protease contribution to serum protein hydrolysis [5]. Consequently, the RT-PCR data presented herein, combined with the detection of CtsD by ELISA, additionally demonstrate that aspartic protease activity is also induced in the presence of serum proteins. Thus, we suggest that future studies, on the elucidation of serum factors which may induce protease secretion by $A$. fumigatus, should include determination of protease activity over a wide $\mathrm{pH}$ range.

\section{Expression of ctsD in A. fumigatus-infected G. mellonella}

Precise quantitation of total RNA (larval and fungal) used for cDNA synthesis was undertaken (data not shown). Furthermore, the presence of genomic $c t s D$ was eliminated by the use of DNase I and confirmed by the amplification of calm cDNA ([23] $348 \mathrm{bp}$; Fig. 6a). G. mellonella calm cDNA is identical in size to that from A. fumigatus and so is apparent in uninfected Galleria. It can be seen from Fig. $6 \mathrm{~b}$ that $c t s D$ expression was evident at $24,48,72$ and $96 \mathrm{~h}$ post-inoculation of $G$. mellonella with A. fumigatus conidia. Moreover, amplicons were subjected to DNA sequence analysis which confirmed they were of fungal origin (data not shown). There was an apparent increase in cts $D$ expression between 24 and $96 \mathrm{~h}$ (Fig. 6b) and a similar pattern of expression was observed for opsB (Fig. 1), although a higher level of expression was evident relative to

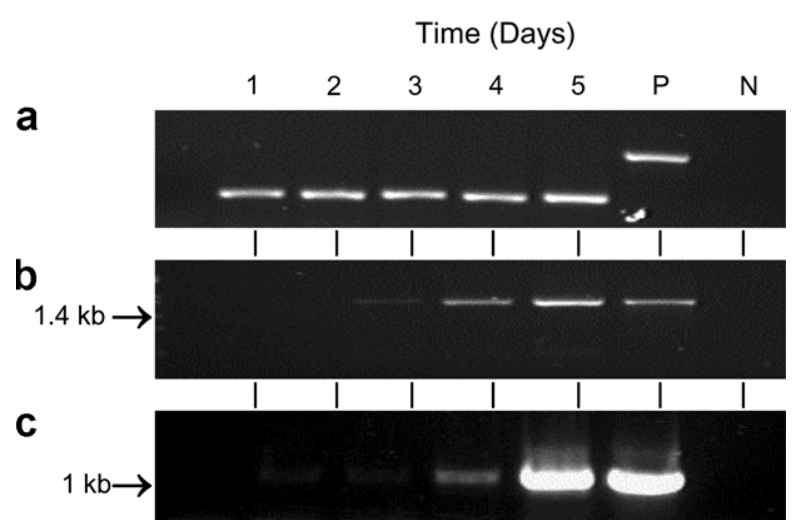

Fig. 6. Infection model studies. (a) RT-PCR analysis of calm control expression [23] in a Galleria mellonella virulence model. (Lane 1) Uninfected Galleria. (Lanes 2-5) 24, 48, 72 and $96 \mathrm{~h}$ post-infection with $A$. fumigatus conidia $\left(2 \times 10^{5}\right)$. (Lane P) calm genomic DNA. (Lane N) Negative control. (b) Expression analysis of ctsD. (Lanes 2-5) ctsD expression in infected Galleria, 24, 48, 72 and $96 \mathrm{~h}$ post-infection with A. fumigatus conidia, respectively. (Lane P) ctsD genomic DNA. (Lane N) Negative control. (c) Expression analysis of ops $B$ (Fig. 1). (Lanes 2-5) ops $B$ expression in infected Galleria, 24, 48, 72 and $96 \mathrm{~h}$ post-infection with $A$. fumigatus conidia, respectively. (Lane P) ops B genomic DNA. (Lane N) Negative control. 


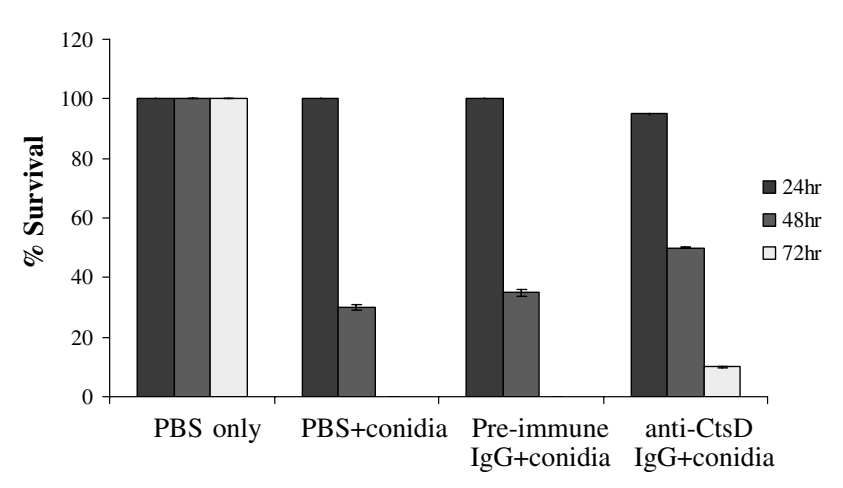

Fig. 7. Determination of virulence potential of CtsD in Galleria mellonella insect model. A statistically significant $(p=0.0004)$ improvement in the survival rate of $G$. mellonella co-inoculated with anti-CtsD IgG $(20 \mu \mathrm{g})$ and $A$. fumigatus conidia $\left(10^{7}\right)$ at $72 \mathrm{~h}$ was observed, relative to co-inoculation with either pre- immune IgG and $A$. fumigatus conidia $\left(10^{7}\right)$, PBS or A. fumigatus conidia $\left(10^{7}\right)$.

cts $D$ (Fig. 6c). While it cannot be entirely excluded that the observed increase in $c t s D$ and $o b s B$ expression, respectively, was due to the larger fungal mass present in the larval material, the consistency of calm cDNA strongly suggests that increased aspartic protease gene expression had occurred. Relevantly, da Silva Ferreira et al. [30] have also detected significantly increased ops $B$ expression in $A$. fumigatus mycelia as part of microarray studies of alteration in global gene expression following exposure to the antifungal drug, voriconazole. Thus, we hypothesise ops $B$ expression occurs in response to either host- or druginduced stress in A. fumigatus and merits future study.

A statistically significant improvement in the survival rate of the Galleria was observed at $72 \mathrm{~h}(p=0.0004)$, following the co-administration of anti-CtsD $\operatorname{IgG}$ and $A$. fumigatus conidia, relative to the administration of $A$. fumigatus conidia alone or conidia with pre-immune $\operatorname{IgG}$ (Fig. 7). This observation further confirms that CtsD expression occurs in A. fumigatus-infected G. mellonella. To our knowledge, one other secreted aspartic protease of $A$. fumigatus has been previously characterised, namely, PEP1 [10] whereas PEP2 has been shown to be associated with the fungal cell wall. Mutants lacking PEP1 showed no alteration in the virulence potential of the fungus. The in vivo virulence potential of other proteases such as MEP and ALP have also been investigated [11,12], with results indicating that no significant difference in virulence was obtained with mutants lacking these proteases. Thus, our data may represent the first identification of an A. fumigatus encoded aspartic protease (CtsD) associated with the fungal virulence, although direct proof will require the generation of a $c t s D$ mutant followed by comparison with $A$. fumigatus wild-type pathogenicity. The use of G. mellonella to assess fungal virulence is attracting ever-increasing attention. Small and Bidochka [31] have evaluated the pattern of Metarhizium anisophilae $\mathrm{prl}$ gene expression in $G$. mellonella and others [32] have ascertained the virulence attenuation of methylcitrate synthetase mutants of $A$. fumigatus. The Galleria model system has also been used to determine the unaltered virulence of $A$. fumigatus mutants [33]. However, the use of antibody administration to diminish the effects of potential virulence factors has not been previously reported and offers an alternative approach for the use of the G. mellonella system in virulence assessment studies.

In conclusion, a novel aspartic protease, CtsD, has been identified and characterised from the pathogenic fungus, Aspergillus fumigatus. CtsD expression was repressed under nutrient-rich culture conditions. Expression of ctsD was detected in Galleria mellonella insect larvae inoculated with $A$. fumigatus and anti-CtsD IgG exhibited a statistically significant protective effect against fungal infection in the insect model system. These data represent the first identification of this novel aspartic protease and suggest possible functions for the enzyme in $A$. fumigatus.

\section{Acknowledgments}

Imelda Vickers was a recipient of an IRCSET PhD studentship. This work was also funded under the Irish Government Programme for Research in Third Level Institutions (PRTLI, Cycle 3). Preliminary sequence data were obtained from The Institute for Genomic Research website at http://www.tigr.org. Sequencing of Aspergillus fumigatus was funded by the National Institute of Allergy and Infectious Disease U01 AI 48830 to David Denning and William Nierman, the Wellcome Trust, and Fondo de Investicagiones Sanitarias. Mass spectrometry facilities were funded by the Health Research Board.

\section{References}

[1] F. Tekaia, J.P. Latge, Aspergillus fumigatus: saprophyte or pathogen? Curr. Opin. Microbiol. 8 (2005) 385-392.

[2] D.B. Archer, P.S. Dyer, From genomics to post-genomics in Aspergillus, Curr. Opin. Microbiol. 7 (2004) 499-504.

[3] W.C. Nierman, A. Pain, M.J. Anderson, J.R. Wortman, H.S. Kim, J. Arroyo, et al., Genomic sequence of the pathogenic and allergenic filamentous fungus Aspergillus fumigatus, Nature 438 (2005) 11511156.

[4] U. Reichard, M. Monod, F. Odds, R. Ruchel, Virulence of an aspergillopepsin-deficient mutant of Aspergillus fumigatus and evidence for another aspartic proteinase linked to the fungal cell wall, J. Med. Vet. Mycol. 35 (1997) 189-196.

[5] A.H. Gifford, J.R. Klippenstein, M.M. Moore, Serum stimulates growth of and proteinase secretion by Aspergillus fumigatus, Infect. Immun. 70 (2002) 19-26.

[6] J.R. Naglik, S.J. Challacombe, B. Hube, Candida albicans secreted aspartyl proteinases in virulence and pathogenesis, Microbiol. Mol. Biol. Rev. 67 (2003) 400-428.

[7] U. Reichard, S. Buttner, H. Eiffert, F. Staib, R. Ruchel, Purification and characterisation of an extracellular serine proteinase from Aspergillus fumigatus and its detection in tissue, J. Med. Microbiol. 33 (1990) 243-251.

[8] J. Kunert, P. Kopecek, Multiple forms of the serine protease Alp of Aspergillus fumigatus, Mycoses 43 (2000) 339-347.

[9] M. Monod, S. Paris, D. Sanglard, K. Jaton-Ogay, J. Bille, J.P. Latge, Isolation and characterization of a secreted metalloprotease of Aspergillus fumigatus, Infect. Immun. 61 (1993) 4099-4104. 
[10] U. Reichard, H. Eiffert, R. Ruchel, Purification and characterization of an extracellular aspartic proteinase from Aspergillus fumigatus, J. Med. Vet. Mycol. 32 (1994) 427-436.

[11] K. Jaton-Ogay, S. Paris, M. Huerre, M. Quadroni, R. Falchetto, G. Togni, J.P. Latge, M. Monod, Cloning and disruption of the gene encoding an extracellular metalloprotease of Aspergillus fumigatus, Mol. Microbiol. 14 (1994) 917-928.

[12] M. Monod, S. Paris, J. Sarfati, K. Jaton-Ogay, P. Ave, J.P. Latge, Virulence of alkaline protease-deficient mutants of Aspergillus fumigatus, FEMS Microbiol. Lett. 106 (1993) 39-46.

[13] M. Kretschmar, B. Hube, T. Bertsch, D. Sanglard, R. Merker, M. Schroder, H. Hof, T. Nichterlein, Germ tubes and proteinase activity contribute to virulence of Candida albicans in murine peritonitis, Infect. Immun. 67 (1999) 6637-6642.

[14] M. Schaller, H.C. Korting, W. Schafer, J. Bastert, W. Chen, B. Hube, Secreted aspartic proteinase (Sap) activity contributes to tissue damage in a model of human oral candidosis, Mol. Microbiol. 34 (1999) $169-180$

[15] U. Reichard, G.T. Cole, T.W. Hill, R. Ruchel, M. Monod, Molecular characterization and influence on fungal development of ALP2, a novel serine proteinase from Aspergillus fumigatus, Int. J. Med. Microbiol. 290 (2000) 549-558.

[16] R. Crameri, Epidemiology and molecular basis of the involvement of Aspergillus fumigatus in allergic diseases, Contrib. Microbiol. 2 (1999) 44-56.

[17] M. Monod, S. Capoccia, B. Lechenne, C. Zaugg, M. Holdom, O. Jousson, Secreted proteases from pathogenic fungi, Int. J. Med. Microbiol. 292 (2002) 405-419.

[18] N.D. Rawlings, F.R. Morton, A.J. Barrett, MEROPS: the peptidase database, Nucleic Acids Res. 34 (2006) D270-D272.

[19] C. Neville, A. Murphy, K. Kavanagh, S. Doyle, A 4'-phosphopantetheinyl transferase mediates non-ribosomal peptide synthetase activation in Aspergillus fumigatus, ChemBioChem 6 (2005) 679-685.

[20] M. Takahashi, T. Sekine, N. Kuba, S. Nakamori, M. Yasuda, H. Takagi, The production of recombinant APRP, an alkaline protease derived from Bacillus pumilus TYO-67, by in vitro refolding of pro-enzyme fixed on a solid surface, J. Biochem. (Tokyo). 136 (2004) 549-556.

[21] U. Reichard, G.T. Cole, R. Ruchel, M. Monod, Molecular cloning and targeted deletion of PEP2 which encodes a novel aspartic proteinase from Aspergillus fumigatus, Int. J. Med. Microbiol. 290 (2000) 85-96.
[22] T. Kuriyama, D.W. Williams, M.A. Lewis, In vitro secreted aspartyl proteinase activity of Candida albicans isolated from oral diseases and healthy oral cavities, Oral Microbiol. Immunol. 18 (2003) 405-407.

[23] C. Burns, R. Geraghty, C. Neville, A. Murphy, K. Kavanagh, S. Doyle, Identification, cloning and functional expression of three glutathione transferase genes from Aspergillus fumigatus, Fungal Genet. Biol. 42 (2005) 319-327.

[24] K. Kavanagh, E.P. Reeves, Exploiting the potential of insects for in vivo pathogenicity testing of microbial pathogens, FEMS Microbiol. Rev. 28 (2004) 101-112.

[25] A. Journet, A. Chapel, S. Jehan, C. Adessi, H. Freeze, G. Klein, J. Garin, Characterization of Dictyostelium discoideum cathepsin D, J. Cell Sci. 112 (1999) 3833-3843.

[26] G. Jarai, D. Kirchherr, F.P. Buxton, Cloning and characterization of the pepD gene of Aspergillus niger which codes for a subtilisin-like protease, Gene 139 (1994) 51-57.

[27] U. Reichard, B. Lechenne, A.R. Asif, F. Streit, E. Grouzmann, O. Jousson, M. Monod, Sedolisins, a new class of secreted proteases from Aspergillus fumigatus with endoprotease or tripeptidyl-peptidase activity at acidic pHs, Appl. Environ. Microbiol. 72 (2006) 1739-1748.

[28] G. Richo, G.E. Conner, Proteolytic activation of human procathepsin D, Adv. Exp. Med. Biol. 306 (1991) 289-296.

[29] M. Schwienbacher, M. Weig, S. Thies, J.T. Regula, J. Heesemann, F. Ebel, Analysis of the major proteins secreted by the human opportunistic pathogen Aspergillus fumigatus under in vitro conditions, Med. Mycol. 43 (2005) 623-630.

[30] M.E. da Silva Ferreira, I. Malavazi, M. Savoldi, A.A. Brakhage, M.H. Goldman, H.S. Kim, W.C. Nierman, G.H. Goldman, Transcriptome analysis of Aspergillus fumigatus exposed to voriconazole, Curr. Genet. 50 (2006) 32-44.

[31] C.L. Small, M.J. Bidochka, Up-regulation of Prl, a subtilisin-like protease, during conidiation in the insect pathogen Metarhizium anisopliae, Mycol. Res. 109 (2005) 307-313.

[32] C. Maerker, M. Rohde, A.A. Brakhage, M. Brock, Methylcitrate synthase from Aspergillus fumigatus, FEBS J. 272 (2005) 3615-3630.

[33] S. Krappmann, C. Sasse, G.H. Braus, Gene targeting in Aspergillus fumigatus by homologous recombination is facilitated in a nonhomologous end-joining-deficient genetic background, Eukaryot. Cell 5 (2006) 212-215. 